\title{
Knowledge Based Strategies for Parametric Design in Architecture
}

\author{
$>$ Roland Hudson \\ Universidad de Los Andes, Colombia \\ r.hudson@uniandes.edu.co
}

\begin{abstract}
Design professionals in the construction industry widely use the term "parametric". Despite this there is a paucity of applied guidelines for developing parametric models. It is possible to attend workshops and learn the mechanics of a specific applications and access online repositories of 'design patterns', but the cognitive process of application in practice has received little recent attention. Analysis and experience of practice indicates that acquisition of new knowledge and capture of existing knowledge are the basis for all parametric tasks. This paper exposes a deeper understanding of the role of the parametric designer and proposes an applied strategic framework.
\end{abstract}

Keywords: Parametric design; Design strategies; Computational thinking

\section{Introduction}

Desktop parametric tools have been available to construction professionals for almost a decade. Creating a parametric model typically requires the organization of a series of nodes and connections to in a visual graph. Adjusting variables in the graph then enables exploration of a variety of possible candidates that lie within a solution space defined by the graph. Defining this graph structure involves externalizing the understanding of the design task. The parametric model describes and represents the parametric designer's knowledge of the task. In architectural design initially the task is ill-structured, the goals are vague and the means of achieving them unknown (Rowe 1987, Simon 1973). How does the parametric designer know they have a good understanding of the design task? If the designer cannot describe the task sufficiently the graph structure may lead to an exploration of an irrelevant solution space.

Seeking and externalizing knowledge of the problem to define the graph is the biggest challenge facing the parametric designer (Aish and Woodbury 2005, Woodbury and Marques 2006), for this reason a strategic approach is required. This paper proposes a cyclic, strategic approach to applied parametric design that reinforces the recognized importance of knowledge in design (Chandrasekaran 1990, Motta 1996). A strategy is a high level plan to achieve one or more goals under conditions of uncertainty. The strategies proposed here lead to execution of design tactics to achieve specific objectives.

The framework for this strategic approach draws on doctoral research (Hudson 2010) that examined design theory cross-referenced to published case studies and a series of participant-observer case studies. Analysis of these sources indicates a task structure where parametric design is generalized as mutually dependant, cyclic, strategic phases (Figure 1). The need to gain initial knowledge of the problem defines the first phase which is closely linked to a model construction phase. The model is then used to investigate design options. Critique of the options will indicate changes required of the model or adjustments to the way that the problem has been understood. The final phase concerns sharing information and construction documentation and this is linked back to the model construction phase and back to a description of the problem.

Deeper examination of this task structure reveals that the primary task involves understanding the problem, which requires capture of existing knowledge or acquisition of new knowledge. Knowledge development and knowledge capture are two primary strategic approaches to parametric design and practical examples are given.

\section{Selected applied case studies}

The objective of the research documented in this paper was to develop an understanding of applied parametric design. To form a theoretical point of departure a broad literature review of design theory and specifically that related to parametric design was undertaken. This basis was expanded through review of a series of published case studies. Thirdly in order to gain a practical perspective the author was directly involved in applying commercially available parametric software to design scenarios in industry and in training professionals and students. 


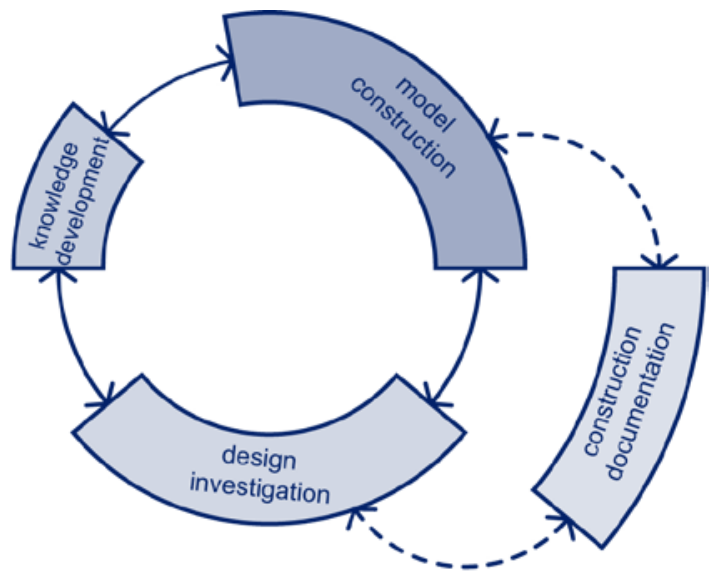

\section{Task Structure Approach}

Primary tasks of the parametric designer

The initial research goal was to map a full description of the parametric design task and align these with practical examples. Each of the practical applications were mapped onto a diagram that represents the task structure for a parametric designer. Using the literature review and analysis of published case studies, the primary tasks in the structure were identified as:

- Create model

- Develop reusable models

- Design Investigation

- Construction documentation

This task structure was broken into sub-tasks and procedures

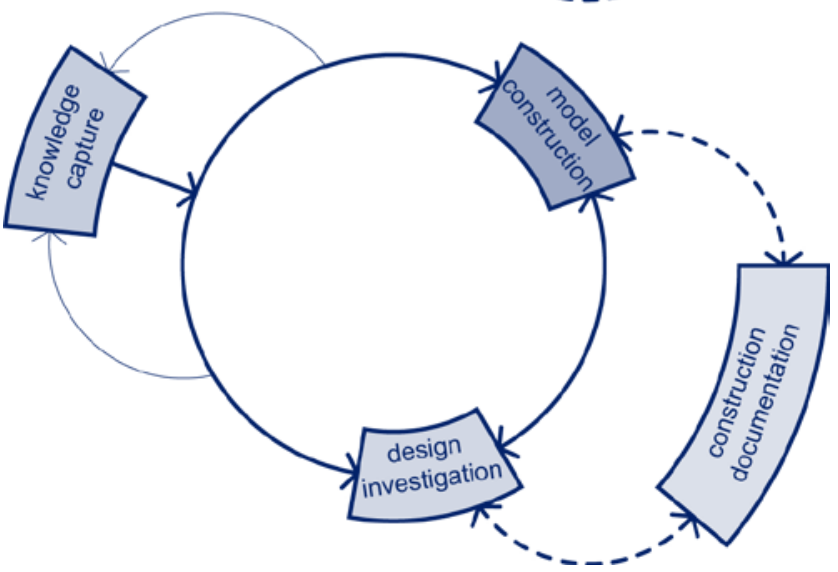
in order to enable effective navigation. Tasks, sub-tasks and procedures from this structure are identified by italics in this section.

Some contradictions were observed between the published case studies and the theoretic literature review. Published case studies confirmed the broad definition of parametric tasks provided by the theoretical literature. Few practical examples of the sub-tasks and procedures proposed by theorists were provided. Instead the published case studies suggested further sub-tasks and procedures through descriptions of specific projects.

The first theoretical parametric sub-task was develop a problem description. Published case studies confirmed the create model task but suggested alternative sub-tasks. They were concerned with translating ideas developed by non-parametric designers into parametric models.

The literature review proposed the sub-task explore design

Figure 1: Knowledge development (top) and knowledge capture (bottom) are cyclic strategies for parametric design. Each determines and is determined by varying relationships to model development, design investigation and construction documentation. Created by R. Hudson.

The author's practical experience that informs this study includes the AVIVA Stadium in Dublin with Architects Populous. The author was the sole parametric modeler for the architects developing a parametric model that interfaced with the structural analysis model, was used for development and manufacture of the cladding system and as a construction coordination model. Also for Populous the author developed a Stadium seating bowl modeler which provides a significant reference for this work.

A series of other projects were undertaken and analysed. In particular the author contributed to the development of one tower for Ian Simpson architects and two towers RMJM/Newtecnic (referred to as Blackfriar's, Moscow and Gazprom herein). Modeling strategy and technical workshops at Ian Simpsons and Rambol provided the opportunity to study and consult in both architectural and structural engineering offices. The author was part of the team that rationalized the geometric definition and undertook structural analysis of the biomes in Singapore Gardens in the Bay for Atelier One and Wilkinson Eyre. Introductory training courses in parametric design were given at over 30 European academic institutions from 2005-2009 providing valuable opportunities observe the responses of students and further develop ideas outlined in this paper. space. This involved seeking a design from a very large range of possibilities. Published case studies described the same sub-task as making small changes to a design by generating and testing ideas based on performance or construction logic.

\section{Contribution of practical studies}

The AVIVA Stadium provided practical examples of sub-tasks and procedures that had been described in the theoretical literature as part of the create model task. In particular AVIVA illustrated how parametric design could be used to develop problem descriptions. AVIVA illustrated sub-tasks related to design investigation. Exploration was started by assigning initial values extracted from a non-parametric model to a parametric definition. The design of the AVIVA facade showed how propose-critique-modify could be applied as a design method.

The seating bowl modeller (SBM) project identified an additional top level task: develop reusable models. This shared similar initial sub-tasks and procedures with the create model task. The SBM extends these approaches to include developing systems for use by others and the need to accurately capture more extensive functionality. SBM identified the sub-task knowledge capture which was undertaken using a method of propose-critique-modify. The importance of re-structuring models was demonstrated during the development process of the SBM, and suggested that this procedure could benefit all model development tasks. The reusable model is 
for use by others in the practice so the SBM identified a further sub-task implement in practice.

The SBM provided a practical example of assigning initial values by identifying parameter ranges from verbal descriptions given by experts. These ranges together with predefined sets of parameters illustrated how in practice it was possible to reduce solution space with amplifiers. The SBM case study defines a strategy for developing models for placing in libraries as reusable models. Such models should be accompanied by a meta-library of parameter sets that form the starting point of new designs.

The applied case studies provided supporting examples for various tasks, sub-tasks and procedures. The tower projects illustrated how use of memory could be used to initiate model construction. The author's parametric design experience was observed to form the basis for the geometric method used for each of the tower models. One tower design case study suggested that this previous experience could also formally define a generic method for tower design. This generic model was used as a starting point for a new model, illustrating the procedure case retrieval. A generic structural tower system demonstrated a process that focused on defining parameters rather than shape.

Analysis of the further case studies provided additional examples of rationalisation and specifically post-rationalisation that was achieved through matching geometry by reducing to simple elements and seeking flat panel solutions. The implications of implementing a parametric model with a purely code based representation was illustrated by work on the two Biome structures in Singapore Gardens by the Bay. In particular the code for the biomes provided a counter point to the fragmented and hierarchical control systems observed in the other towers. The biomes' abstract mathematical geometry method statement contrasted the more procedural geometric method statements used by Foster and Partner's Specialist Modelling Group. The limits of sharing geometric information as a series of instructions written in plain English rather than code were exposed. One of the tower projects used a scripted representation to automate design exploration and as such demonstrates the process reduce solution space using amplifiers. Use of multiple representations in the explore design space task was illustrated by two of the projects which automatically generated models for setting up structural analysis. AVIVA the Moscow tower demonstrated evaluation of full-scale construction logic. Assessment of aesthetics directed a generate-and-test procedure for design investigation stages of Blackfriar's Tower in London.

\section{Analysis of task structure}

The task structure itself represents a significant contribution to the limited information surrounding the practical approaches to parametric design. Deeper analysis of the task structure indicates that the parametric design process can be generalised as a series of mutually dependant phases and strategies are proposed for each. Primarily this analysis indicates that the process is intensive in terms of requiring the capture and development of the parametric designers existing knowledge. The need to gain an understanding of the problem defines the first phase, this is closely linked to a model construction phase. The model is then used to investigate options. Critique of the model itself investigation will indicate changes required of the model or adjustments to the way that the problem has been understood. The final phase, which is beyond the scope of this paper, concerns sharing information for construction documentation and this too is closely linked to the model construction phase.

Two approaches for gaining an understanding of the problem are identified; knowledge development and knowledge capture. Knowledge development is required when a problem description needs to be defined. Knowledge capture is employed where the problem is already known and the aim is to parameterise it. This approach is common to the sub-tasks capture design intent and develop reusable tools. Emphasis in capture design intent is on matching existing geometry. Whereas for develop reusable tools the focus is instead making the captured knowledge usable and accessible to others.

The task structure indicates a series of common principles for constructing models. These principles form the core strategic approach. Model construction is directed by knowledge of the problem which has been either developed or captured. The sub-task here is to externalise the ideas defined by knowledge of the problem. This involves proposing a model structure, critiquing it and then modifying or restructuring both the knowledge of the problem and the model itself. Knowledge of the problem and the model construction are therefore interdependent. Once a model has been defined it can be used to undertake design investigation. This will test the knowledge of the problem, the model construction and the designed artefact.

In order to conduct the investigation the model must be controllable and produce results that satisfy the functional requirements defined by the problem. The results of this test can then feedback into the development and capture of the problem knowledge, the model construction and the design. The task structure indicates investigation procedures corresponding with early design investigations and later refining of details. Early design investigations are broader in the scope of options explored and relate more closely to the knowledge development approach. Refining details is a design investigation task that occurs with a later stage of design where the problem description is known or has been well developed.

The final strategic approach involves construction documentation. This too is closely linked to model construction, and determines the extent of the investigation stages. The task structure indicates that the representations required for contractors are the primary consideration. The extraction of these will need to be coordinated with the model construction. If extracting information for construction has been integrated into the design process, and tested in good time, design investigations can take place later into the project time-line. Opportunities exist here for extending the role of the parametric designer. By sharing the model or the parametric skills it may be possible to add efficiency to the contractor's workflow.

\section{Knowledge Development}

Knowledge development strategy should be implemented when a problem description is undefined or partially defined. It is highly 
dependent on both model construction and design investigation phases. The link between these parts is essential, developing a problem description using a parametric process requires feedback from design investigation as soon as a model has been developed, and a model cannot be developed until some problem description has been established. The feedback informs revisions to both problem description and the structure of the model.

The dependency on feedback from aspects of the model construction and design investigation strategies means that knowledge development requires models to be established quickly so the iterative process of developing the model can begin. knowledge development, model construction and design Investigation need to be tackled simultaneously and constantly re-evaluated and revisited as the process progresses and design exploration is undertaken. The problem description develops through the process of defining models by externalising ideas and then assessing both the model function and its output. The following considerations are proposed for this strategic approach:

- Use when a problem description doesn't exist or is partial.

- Implement model construction and design Investigation simultaneously.

- $\quad$ Re-evaluate the problem description after assessing model construction and design Investigation.

- Identify parameters, functions and constraints.

- Make assumptions and test them.

- Fragment the problem.

\section{Knowledge Capture}

Knowledge capture is intended for situations where a problem description has already been developed or where a reusable tool is required. It should be applied when the intention is to parameterize an existing design process and undertake an exploration with the goal of refining the existing design rather than redefining it. In a similar way to the knowledge development strategy, knowledge capture is dependent on model construction and design investigation. Feedback from the model construction and design investigation is not used to redefine or substantially restructure the model or the definition problem description but to check that the existing design intent or design process has been correctly captured.

Checking the knowledge captured is correct is particularly important if the model is to be used by several people. When implemented in practice, user feedback will indicate how well the existing knowledge has been understood and captured and the quality of the model and control mechanisms. Whether capturing an existing design idea or developing reusable tools with knowledge capture the following considerations are proposed:

- Use when translating a non-parametric description to a parametric description.

- Anticipate differences in terminology.

- Construct initial models based on translation attempts.

- Assess proposed models to check design intent has translated.

- Rebuild or revise models.

- Anticipate cyclic development of model.

\section{Model Construction}

Model Construction defines a series of core principles for constructing parametric models. It is mutually dependant on other strategies that precede and follow it as it is both informed by and informs how these develop. Given some knowledge or description of the problem it is possible to begin to construct a model and externalise ideas. This is about capturing expertise, experience and constraints (Kilian, 2006). It involves using software to explicitly develop relationships between objects. If those relationships or objects do not exist then bespoke tools must be constructed (Aish \& Woodbury, 2005). It is proposed that the strategy requires consideration of the following points:

- Get initial ideas from a library, memory or retrieve a previous case.

- Treat early models as disposable.

- Anticipate incremental development.

- Propose critique and modify successive models.

- Think first of parameters and secondly shape.

- Consider model composition.

- Fragment the model and reassess as the project progresses.

- Assess design team size and skill levels.

- Identify key parameters.

- Implement a control hierarchy.

\section{Design Investigation}

Exploration or investigation has been described as the primary task for the designer (Kilian, 2006). Fundamentally this is about generating a design and testing it against the requirements defined by the problem description. design investigation defines a series of strategic principles for investigating design alternatives. Design investigation is closely linked to the earlier strategic phases. It tests if the problem description is correct, if the model performs in the required way and if the design satisfies the problem requirements. design Investigation should be undertaken with the previous stages simultaneously or at least as soon as sufficient knowledge of the problem has been developed or captured to construct a model. Feedback from assessment is passed back to the model creation phase and adjustments made to the model. When undertaking design Investigation the following points should be considered:

- Expect extensive investigation if preceded by knowledge development.

- Expect refining of parameters if preceded by knowledge capture.

- Assign initial parameter values.

- Evaluate the design.

- Implement amplifiers to aid exploration of parameters.

- Identify representations for assessment and incorporate with model construction.

- Following assessment return to knowledge development and model construction and update.

\section{Conclusion}

These strategies challenge areas of theoretical thinking and the observed practical norm of parametric design where models are only 
created once a problem description is fully defined (Burry 2003, Maher 2006). In line with other theoretical views (Schon 1991, Chandrasekaran 1990), this paper proposes that design problem descriptions can develop dynamically. When knowledge development and knowledge capture are pursued as primary strategic objectives, parametric modeling enables broader understanding of the design task.

Observations of introductory training courses in parametric design conducted by the author support the original claims of this paper. Designers experienced in modelling with non-parametric software find the concepts required for parametric design too abstract. These difficulties were one of the original motivations for undertaking this research. In the course of this research the author has developed a short training curriculum which includes some of the findings reported in this paper. This involves participants tackling a practical building design task which reflects some of the strategic approaches described. With careful and deliberate explanation this can illustrate the abstract concepts of parametric design and demonstrate the practical benefits of implementing these.

\section{Acknowledgments}

University of Bath, Bentley Systems, EPSRC UK, Populous, Ian Simpsons Architects, Rambol, Atelier One, Newtecnic Chris Williams.

\section{References}

Aish, R., \& Woodbury, R. (2005) .Multi level interaction in parametric design, Lecture notes in computer science, 3638, 151-162.
Burry, M. (2003). Between intuition and process: Parametric design and rapid prototyping. In B. Kolarevic, (Ed), Architecture in the digital age - design and manufacturing. (pp 147-162). Abingdon: Taylor and Francis,

Chandrasekaran, B. (1990). Design problem solving: A task analysis. AI magazine, 11(4), 59-71.

Hudson, R. (2010). Strategies for parametric design in architecture. Ph.D. thesis, University of Bath.

Killian, A. (2005). Design exploration through bidirectional modeling of constraints. Ph.D. thesis, Massachusetts Institute of Technology.

Maher, A. (2006). The parametric bridge. Selfridges Birmingham UK. In A. Chaszar (Ed), Blurring the lines. (pp 76-81). London, Wiley.

Motta, E., \& Zdrahal, Z. (1996). Parametric design problem solving. Open University, doi=10.1.1.26.8464.

Rowe, P. G. (1987). Design thinking. Cambridge Massachusetts: The MIT Press.

Schon, D.A. (1991). The reflective practitioner: how professionals think in action. Aldershot: Ashgate,

Simon, H.A. (1973). The structure of ill-structured problems. Artificial intelligence, 4(3), 181-201.

Woodbury, R., \& Marques, D. (2006). Using rule based selection to support change in parametric cad models, Lecture notes in computer science, 4073, 230-235. 